\title{
Conformal Hemi-Slant Riemannian Maps
}

\author{
Şener Yanan (10* \\ Adıyaman University, Faculty of Arts and Sciences, Department of Mathematics \\ Adıyaman, Türkiye
}

Received: 07 December 2021

Accepted: 20 January 2022

\begin{abstract}
In this study, we define conformal hemi-slant Riemannian maps from an almost Hermitian manifold to a Riemannian manifold as a generalization of conformal anti-invariant Riemannian maps, conformal semi-invariant Riemannian maps and conformal slant Riemannian maps. Then, we obtain integrability conditions for certain distributions which are included in the notion of hemi-slant Riemannian maps and investigate their leaves. Also, we get totally geodesic conditions for this type maps. Lastly, we introduce some geometric properties under the notion of pluri-harmonic map.
\end{abstract}

Keywords: Riemannian submersion, Riemannian map, conformal Riemannian map, conformal hemi-slant Riemannian map.

\section{Introduction}

Particularly, the concept of Riemannian submersions [6] and isometric immersions [5] were studied by Falcitelli and Chen. Then, Riemannian submersions were studied in various types as an antiinvariant, a semi-invariant, a slant and a hemi-slant [16]. Then, this concept generalized to the notion of Riemannian map by Fischer [7]. Riemannian maps between Riemannian manifolds are generalization of isometric immersions and Riemannian submersions. Let $\Phi:\left(M_{1}, g_{1}\right) \longrightarrow\left(M_{2}, g_{2}\right)$ be a smooth map between Riemannian manifolds such that $0<\operatorname{rank} \Phi<\min \left\{\operatorname{dim}\left(M_{1}\right), \operatorname{dim}\left(M_{2}\right)\right\}$. Then, the tangent bundle $T M_{1}$ of $M_{1}$ has the following decomposition:

$$
T M_{1}=\operatorname{ker} \Phi_{*} \oplus\left(\operatorname{ker} \Phi_{*}\right)^{\perp}
$$

Since $\operatorname{rank} \Phi<\min \left\{\operatorname{dim}\left(M_{1}\right), \operatorname{dim}\left(M_{2}\right)\right\}$, always we have $\left(\operatorname{range} \Phi_{\star}\right)^{\perp}$. In this way, tangent bundle $T M_{2}$ of $M_{2}$ has the following decomposition:

$$
T M_{2}=\left(\operatorname{range} \Phi_{*}\right) \oplus\left(\operatorname{range} \Phi_{*}\right)^{\perp} .
$$

A smooth map $\Phi:\left(M_{1}^{m}, g_{1}\right) \longrightarrow\left(M_{2}^{n}, g_{2}\right)$ is called Riemannian map at $p_{1} \in M_{1}$ if the horizontal restriction $\Phi_{* p_{1}}^{h}:\left(\operatorname{ker} \Phi_{* p_{1}}\right)^{\perp} \longrightarrow\left(\operatorname{range} \Phi_{*}\right)$ is a linear isometry. Hence, a Riemannian map *Correspondence: syanan@adiyaman.edu.tr 2020 AMS Mathematics Subject Classification: 53C15, 53C55

This article is licensed under a Creative Commons Attribution 4.0 International License.

Also, it has been published considering the Research and Publication Ethics. 57 
satisfies the equation

$$
g_{1}(X, Y)=g_{2}\left(\Phi_{\star}(X), \Phi_{*}(Y)\right)
$$

for $X, Y \in \Gamma\left(\left(\operatorname{ker} \Phi_{*}\right)^{\perp}\right)$. So that isometric immersions and Riemannian submersions are particular Riemannian maps, respectively, with $\operatorname{ker} \Phi_{*}=\{0\}$ and $\left(\operatorname{range} \Phi_{*}\right)^{\perp}=\{0\}[6]$. An important application field of Riemannian maps is the eikonal equation. It acts as a bridge between geometric optics and physical optics. Also, Riemannian maps and their applications studied by Garcia-Rio and Kupeli in semi-Riemannian geometry [8].

Moreover, Şahin introduced any other types of Riemannian maps [13-16]. In further studies, in particular Akyol, Şahin and Yanan searched this type submersions [1-3] and Riemannian maps $[18-21]$ under conformality case, see also [9]. We say that $\Phi:\left(M^{m}, g_{M}\right) \longrightarrow\left(N^{n}, g_{N}\right)$ is a conformal Riemannian map at $p \in M$ if $0<\operatorname{rank} \Phi_{* p} \leq \min \{m, n\}$ and $\Phi_{* p}$ maps the horizontal space $\left(k e r\left(\Phi_{* p}\right)^{\perp}\right)$ conformally onto $\operatorname{range}\left(\Phi_{* p}\right)$, i.e., there exist a number $\lambda^{2}(p) \neq 0$ such that

$$
g_{N}\left(\Phi_{* p}(X), \Phi_{* p}(Y)\right)=\lambda^{2}(p) g_{M}(X, Y)
$$

for $X, Y \in \Gamma\left(\left(k e r\left(\Phi_{* p}\right)^{\perp}\right)\right.$. Also, $\Phi$ is called conformal Riemannian if $\Phi$ is conformal Riemannian at each $p \in M[17]$.

An even-dimensional Riemannian manifold $\left(M, g_{M}, J\right)$ is called an almost Hermitian manifold if there exists a tensor field $J$ of type $(1,1)$ on $M$ such that $J^{2}=-I$ where $I$ denotes the identity transformation of $T M$ and

$$
g_{M}(X, Y)=g_{M}(J X, J Y), \forall X, Y \in \Gamma(T M) .
$$

Let $\left(M, g_{M}, J\right)$ is an almost Hermitian manifold and its Levi-Civita connection is $\nabla$ with respect to $g_{M}$. If $J$ is parallel with respect to $\nabla$, i.e.,

$$
\left(\nabla_{X} J\right) Y=0
$$

we say $M$ is a Kähler manifold [22].

Therefore, in Section 2; we present background concepts to be used in this paper. In Section 3; we study conformal hemi-slant Riemannian maps from almost Hermitian manifolds to Riemannian manifolds as a generalization of conformal semi-invariant Riemannian maps and conformal slant Riemannian maps. In Section 4; we use the concept of pluriharmonicity to introduce geometric properties.

\section{Preliminaries}

In this section, we give several definitions and results to be used throughout the study for conformal hemi-slant Riemannian maps. Let $\Phi:\left(M, g_{M}\right) \longrightarrow\left(N, g_{N}\right)$ be a smooth map between Riemannian 
manifolds. The second fundamental form of $\Phi$ is defined by

$$
\left(\nabla \Phi_{*}\right)(X, Y)=\stackrel{N}{\nabla_{X}^{\Phi}} \Phi_{*}(Y)-\Phi_{*}\left(\nabla_{X}^{M} Y\right)
$$

for $X, Y \in \Gamma(T M)$. The second fundamental form $\nabla \Phi_{*}$ is symmetric [10].

Then, we define O'Neill's tensor fields $\mathcal{T}$ and $\mathcal{A}$ for Riemannian submersions as

$$
\begin{aligned}
& \mathcal{A}_{X} Y=h \stackrel{M}{\nabla}_{h X} v Y+v \stackrel{M}{\nabla}_{h X} h Y, \\
& \mathcal{T}_{X} Y=h \stackrel{M}{\nabla} v X v Y+v \stackrel{M}{\nabla}_{v X} h Y
\end{aligned}
$$

for $X, Y \in \Gamma(T M)$ with the Levi-Civita connection $\stackrel{M}{\nabla}$ of $g_{M}$ [12]. As usual, we denote by $v$ and $h$ the projections on the vertical distribution $\operatorname{ker} \Phi_{*}$ and the horizontal distribution $\left(\operatorname{ker} \Phi_{*}\right)^{\perp}$, respectively. For any $X \in \Gamma(T M), \mathcal{T}_{X}$ and $\mathcal{A}_{X}$ are skew-symmetric operators on $(\Gamma(T M), g)$ reversing the horizontal and the vertical distributions. Also, $\mathcal{T}$ is vertical, $\mathcal{T}_{X}=\mathcal{T}_{v X}$, and $\mathcal{A}$ is horizontal, $\mathcal{A}_{X}=\mathcal{A}_{h X}$. Note that the tensor field $\mathcal{T}$ is symmetric on the vertical distribution [12]. Additionally, from (6) and (7) we have

$$
\begin{aligned}
& \stackrel{M}{\nabla}_{U} V=\mathcal{T}_{U} V+\hat{\nabla}_{U} V, \\
& \stackrel{M}{\nabla}_{U} X=h \nabla_{U} X+\mathcal{T}_{U} X, \\
& \stackrel{M}{\nabla}_{X} V=\mathcal{A}_{X} V+v \nabla_{X} V, \\
& M_{X} Y=h \nabla_{X} Y+\mathcal{A}_{X} Y
\end{aligned}
$$

for $X, Y \in \Gamma\left(\left(\operatorname{ker} \Phi_{*}\right)^{\perp}\right)$ and $U, V \in \Gamma\left(\operatorname{ker} \Phi_{*}\right)$, where $\hat{\nabla}_{U} V=\stackrel{M}{v} \nabla_{U} V[6]$.

If a vector field $X$ on $M$ is related to a vector field $X^{\prime}$ on $N$, we say $X$ is a projectable vector field. If $X$ is both a horizontal and a projectable vector field, we say $X$ is a basic vector field on $M$. From now on, when we mention a horizontal vector field, we always consider a basic vector field [4].

On the other hand, let $\Phi:\left(M^{m}, g_{M}\right) \longrightarrow\left(N^{n}, g_{N}\right)$ be a conformal Riemannian map between Riemannian manifolds. Then, we have

$$
\begin{aligned}
\left.\left(\nabla \Phi_{*}\right)(X, Y)\right|_{\text {range } \Phi_{*}} & =X(\ln \lambda) \Phi_{*}(Y)+Y(\ln \lambda) \Phi_{*}(X) \\
& -g_{M}(X, Y) \Phi_{*}(\operatorname{grad}(\ln \lambda))
\end{aligned}
$$


where $X, Y \in \Gamma\left(\left(k e r \Phi_{*}\right)^{\perp}\right)$. Hence from $(12)$, we obtain $\stackrel{N}{\nabla_{X}^{\Phi}} \Phi_{*}(Y)$ as

$$
\begin{aligned}
\stackrel{N}{\nabla_{X}^{\Phi}} \Phi_{*}(Y) & =\Phi_{*}(h \stackrel{M}{\nabla} X Y)+X(\ln \lambda) \Phi_{*}(Y)+Y(\ln \lambda) \Phi_{*}(X) \\
& -g_{M}(X, Y) \Phi_{*}(\operatorname{grad}(\ln \lambda))+\left(\nabla \Phi_{*}\right)^{\perp}(X, Y),
\end{aligned}
$$

where $\left(\nabla \Phi_{*}\right)^{\perp}(X, Y)$ is the component of $\left(\nabla \Phi_{*}\right)(X, Y)$ on $\left(\operatorname{range} \Phi_{*}\right)^{\perp}$ for $X, Y \in \Gamma\left(\left(k e r \Phi_{*}\right)^{\perp}\right)$ $[18,19]$.

Lastly, a map $\Phi$ from a complex manifold $\left(M, g_{M}, J\right)$ to a Riemannian manifold $\left(N, g_{N}\right)$ is a pluriharmonic map if $\Phi$ satisfies the following equation

$$
\left(\nabla \Phi_{*}\right)(X, Y)+\left(\nabla \Phi_{*}\right)(J X, J Y)=0
$$

for $X, Y \in \Gamma(T M)[11]$.

\section{Conformal Hemi-slant Riemannian Maps}

We define conformal hemi-slant Riemannian maps from almost Hermitian manifolds and give some examples. We examine integrability and totally geodesicity conditions.

Definition 3.1 A conformal Riemannian map $\Phi:\left(M, g_{M}, J\right) \longrightarrow\left(N, g_{N}\right)$ is called a conformal hemi-slant Riemannian map if the vertical distribution ker $\Phi_{*}$ of $\Phi$ admits two orthogonal complementary distributions $\mathcal{D}_{\theta}$ and $\mathcal{D}_{\perp}$ such that $\mathcal{D}_{\theta}$ is slant and $\mathcal{D}_{\perp}$ is anti-invariant, i.e., we have

$$
\operatorname{ker} \Phi_{*}=\mathcal{D}_{\theta} \oplus \mathcal{D}_{\perp}
$$

Hence, the angel $\theta$ is called the hemi-slant angle of the conformal Riemannian map.

Here, if we denote the dimension of $\mathcal{D}_{\theta}$ and $\mathcal{D}_{\perp}$ by $m_{\theta}$ and $m_{\perp}$, respectively, then we get:

i) If $m_{\theta}=0$, then $\Phi$ is a conformal anti-invariant Riemannian map [18].

ii) If $m_{\perp}=0$ and $\theta=0$, then $\Phi$ is a conformal invariant Riemannian map.

iii) If $m_{\perp}=0$ and $\theta \neq 0, \frac{\pi}{2}$, then $\Phi$ is a proper conformal slant Riemannian map [21].

iv) If $\theta=\frac{\pi}{2}$, then $\Phi$ is a conformal anti-invariant Riemannian map.

Now, we give some examples for conformal hemi-slant Riemannian maps.

Example 3.2 Every conformal slant submersion [3] from an almost Hermitian manifold to a Riemannian manifold is a conformal hemi-slant Riemannian map with $\mathcal{D}_{\perp}=\{0\}$ and $\left(\text { range } \Phi_{*}\right)^{\perp}=$ $\{0\}$. 
Example 3.3 Every conformal hemi-slant submersion [9] from an almost Hermitian manifold to a Riemannian manifold is a conformal hemi-slant Riemannian map with $\left(\text { range } \Phi_{*}\right)^{\perp}=\{0\}$.

Example 3.4 Every conformal slant Riemannian map [21] from an almost Hermitian manifold to a Riemannian manifold is a conformal hemi-slant Riemannian map with $\mathcal{D}_{\perp}=\{0\}$.

Example 3.5 Every conformal semi-invariant submersion [2] from an almost Hermitian manifold to a Riemannian manifold is a conformal hemi-slant Riemannian map with $\theta=\frac{\pi}{2}$ and $\left(\operatorname{range} \Phi_{*}\right)^{\perp}=$ $\{0\}$.

Example 3.6 Every conformal semi-invariant Riemannian map [19] from an almost Hermitian manifold to a Riemannian manifold is a conformal hemi-slant Riemannian map with $\theta=\frac{\pi}{2}$.

If $\mathcal{D}_{\perp} \neq\{0\}$ and $\theta \neq 0, \frac{\pi}{2}$, then we say $\Phi$ is a proper conformal hemi-slant Riemannian map. Hence, we give an explicit example to proper case.

Example 3.7 Define a map $\Phi: \mathbb{R}^{8} \longrightarrow \mathbb{R}^{5}$ by

$$
\Phi\left(x_{1}, x_{2}, x_{3}, x_{4}, x_{5}, x_{6}, x_{7}, x_{8}\right)=e\left(x_{2}, x_{3}, \frac{x_{6}+x_{7}}{\sqrt{2}}, x_{8}, 0\right)
$$

with $\theta \in\left(0, \frac{\pi}{2}\right)$. We obtain the horizontal distribution

$$
\left(\operatorname{ker} \Phi_{*}\right)^{\perp}=\left\{Z_{1}=e \frac{\partial}{\partial x_{2}}, Z_{2}=e \frac{\partial}{\partial x_{3}}, Z_{3}=\frac{e}{\sqrt{2}}\left(\frac{\partial}{\partial x_{6}}+\frac{\partial}{\partial x_{7}}\right), Z_{4}=e \frac{\partial}{\partial x_{8}}\right\}
$$

and the vertical distribution

$$
k e r \Phi_{*}=\left\{W_{1}=\frac{\partial}{\partial x_{1}}, W_{2}=\frac{\partial}{\partial x_{4}}, W_{3}=\frac{\partial}{\partial x_{5}}, W_{4}=\frac{\partial}{\partial x_{6}}-\frac{\partial}{\partial x_{7}}\right\},
$$

respectively. If the complex structure of $\mathbb{R}^{8}$ is $J=\left(-a_{2}, a_{1},-a_{4}, a_{3},-a_{6}, a_{5},-a_{8}, a_{7}\right)$, we have

$$
J W_{1}=\frac{1}{e} Z_{1}, \quad J W_{2}=-\frac{1}{e} Z_{2}, \quad J W_{3}=\frac{\sqrt{2}}{2 e} Z_{3}+\frac{1}{2} W_{4}, \quad J W_{4}=-\frac{1}{e} Z_{4}-W_{3}
$$

Hence, we obtain $\mathcal{D}_{\perp}=\operatorname{span}\left\{W_{1}, W_{2}\right\}$ and $\mathcal{D}_{\theta}=\operatorname{span}\left\{W_{3}, W_{4}\right\}$. So, $\Phi$ is a proper conformal hemi-slant Riemannian map with slant angle $\theta=\frac{\pi}{4}, \lambda=e$ and $\operatorname{rank} \Phi=4$.

For any $W \in \Gamma\left(\operatorname{ker} \Phi_{*}\right)$, we get

$$
W=\tilde{P} W+\tilde{Q} W
$$


where $\tilde{P} W \in \Gamma\left(\mathcal{D}_{\theta}\right)$ and $\tilde{Q} W \in \Gamma\left(\mathcal{D}_{\perp}\right)$, and have

$$
J W=\phi W+\psi W
$$

where $\phi W \in \Gamma\left(k e r \Phi_{*}\right)$ and $\psi W \in \Gamma\left(\left(k e r \Phi_{*}\right)^{\perp}\right)$. Lastly, for $Z \in \Gamma\left(\left(k e r \Phi_{*}\right)^{\perp}\right)$, we have

$$
J Z=B Z+C Z
$$

where $B Z \in \Gamma\left(k e r \Phi_{*}\right)$ and $C Z \in \Gamma\left(\left(k e r \Phi_{*}\right)^{\perp}\right)$. Hence, we obtain decomposition of $\left(k e r \Phi_{*}\right)^{\perp}$ as

$$
\left(\operatorname{ker} \Phi_{*}\right)^{\perp}=\psi \mathcal{D}_{\theta} \oplus J \mathcal{D}_{\perp} \oplus \mu
$$

where $\mu$ is the orthogonal complement of $\psi \mathcal{D}_{\theta} \oplus J \mathcal{D}_{\perp}$ and it is invariant under $J$. From equations (16)-(19), we obtain followings:

$$
\phi \mathcal{D}_{\theta}=\mathcal{D}_{\theta}, \quad \phi \mathcal{D}_{\perp}=\{0\}, \quad B \psi \mathcal{D}_{\theta}=\mathcal{D}_{\theta}, \quad B J \mathcal{D}_{\perp}=\mathcal{D}_{\perp}
$$

and

$$
\phi^{2}+B \psi=-I, \quad \psi \phi+C \psi=\{0\}, \quad \phi B+B C=\{0\}, \quad \psi B+C^{2}=-I .
$$

The proof of the next theorem is exactly same with hemi-slant submanifolds like hemi-slant Riemannian maps; see Theorem 3.6 of [15].

Theorem 3.8 Let $\Phi$ be a conformal Riemannian map from an almost Hermitian manifold $\left(M, g_{M}, J\right)$ to a Riemannian manifold $\left(N, g_{N}\right)$. Then, $\Phi$ is a conformal hemi-slant Riemannian map if and only if there exists a constant $\lambda \in[0,1]$ and a distribution $\mathcal{D}$ on ker $\Phi_{*}$ such that

i) $\mathcal{D}=\left\{W \in \Gamma\left(k e r \Phi_{*}\right) \mid \phi^{2} W=\lambda W\right\}$,

ii) we have $\phi W=0$, for any $W \in \Gamma\left(k e r \Phi_{*}\right)$ orthogonal to $\mathcal{D}$.

Further, we have $\lambda=-\cos ^{2} \theta$ where $\theta$ is the slant angle of $\Phi$.

The next expressions are easy to see their validity

$$
\begin{gathered}
g_{M}\left(\phi U_{1}, \phi U_{2}\right)=\cos ^{2} \theta g_{M}\left(U_{1}, U_{2}\right), \\
g_{M}\left(\psi U_{1}, \psi U_{2}\right)=\sin ^{2} \theta g_{M}\left(U_{1}, U_{2}\right)
\end{gathered}
$$

for any $U_{1}, U_{2} \in \Gamma\left(\mathcal{D}_{\theta}\right)$.

Now, we give some integrability conditions for leaf of the distributions. 
Theorem 3.9 Let $\Phi$ be a conformal hemi-slant Riemannian map from a Kähler manifold $\left(M, g_{M}, J\right)$ to a Riemannian manifold $\left(N, g_{N}\right)$. Then, the slant distribution $\mathcal{D}_{\theta}$ is integrable if and only if

$$
\begin{aligned}
\lambda^{2}\left\{g_{M}\left(\mathcal{T}_{U_{1}} J V, \phi U_{2}\right)-g_{M}\left(\mathcal{T}_{U_{2}} J V, \phi U_{1}\right)\right\} & =\stackrel{N}{N} g_{N}\left(\nabla_{U_{2}}^{\Phi} \Phi_{*}(J V)+\Phi_{*}\left(\mathcal{A}_{J V} U_{2}\right), \Phi_{*}\left(\psi U_{1}\right)\right) \\
& -g_{N}\left(\stackrel{N}{\nabla_{U_{1}}^{\Phi}} \Phi_{\star}(J V)+\Phi_{*}\left(\mathcal{A}_{J V} U_{1}\right), \Phi_{*}\left(\psi U_{2}\right)\right)
\end{aligned}
$$

for any $U_{1}, U_{2} \in \Gamma\left(\mathcal{D}_{\theta}\right)$ and $V \in \Gamma\left(\mathcal{D}_{\perp}\right)$.

Proof Since $g_{M}$ is the Kähler metric from (9) and (17), we get

$$
g_{M}\left(\stackrel{M}{\nabla} U_{U_{1}} U_{2}, V\right)=-g_{M}\left(\mathcal{T}_{U_{1}} J V, \phi U_{2}\right)-g_{M}\left(h \stackrel{M}{\nabla}{ }_{U_{1}} J V, \psi U_{2}\right)
$$

for any $U_{1}, U_{2} \in \Gamma\left(\mathcal{D}_{\theta}\right)$ and $V \in \Gamma\left(\mathcal{D}_{\perp}\right)$. Now, using (5) and symmetry condition of $\nabla \Phi_{*}$, we get

$$
\Phi_{\star}\left(h \stackrel{M}{\nabla} U_{1} J V\right)=\stackrel{N}{\nabla_{U_{1}}^{\Phi}} \Phi_{\star}(J V)+\Phi_{\star}\left(\mathcal{A}_{J V} U_{1}\right)
$$

Putting (25) in (24), we have

$$
\begin{aligned}
g_{M}\left(\stackrel{M}{\nabla} U_{1} U_{2}, V\right) & =-g_{M}\left(\mathcal{T}_{U_{1}} J V, \phi U_{2}\right) \\
& -\frac{1}{\lambda^{2}} g_{N}\left(\nabla_{U_{1}}^{N} \Phi_{*}(J V)+\Phi_{*}\left(\mathcal{A}_{J V} U_{1}\right), \Phi_{\star}\left(\psi U_{2}\right)\right) .
\end{aligned}
$$

Lastly, changing the roles of $U_{1}$ and $U_{2}$ in (26) we obtain the proof.

The integrability condition of $\mathcal{D}_{\perp}$ is the same with Theorem 3.8 in [15]. Note that, always the distribution $\operatorname{ker} \Phi_{*}$ is integrable. Then, we have the following.

Theorem 3.10 Let $\Phi$ be a conformal hemi-slant Riemannian map from a Kähler manifold $\left(M, g_{M}, J\right)$ to a Riemannian manifold $\left(N, g_{N}\right)$. Then, the horizontal distribution $\left(k e r \Phi_{*}\right)^{\perp}$ is integrable if and only if

i)

$$
\begin{aligned}
& g_{N}\left(\left(\nabla \Phi_{*}\right)\left(Z_{2}, B Z_{1}\right)-\left(\nabla \Phi_{*}\right)\left(Z_{1}, B Z_{2}\right)+\stackrel{N}{\nabla_{Z_{1}}^{\Phi}} \Phi_{*}\left(C Z_{2}\right)-\nabla_{Z_{2}}^{\Phi} \Phi_{*}\left(C Z_{1}\right), \Phi_{*}(\psi U)\right) \\
& =\lambda^{2}\left\{g_{M}\left(v \stackrel{M}{\nabla} Z_{1} B Z_{2}+\mathcal{A}_{Z_{1}} C Z_{2}-v \stackrel{M}{\nabla} Z_{2} B Z_{1}-\mathcal{A}_{Z_{2}} C Z_{1}, \phi U\right)\right. \\
& -Z_{1}(\ln \lambda) g_{M}\left(C Z_{2}, \psi U\right)-C Z_{2}(\ln \lambda) g_{M}\left(Z_{1}, \psi U\right)+Z_{2}(\ln \lambda) g_{M}\left(C Z_{1}, \psi U\right) \\
& \left.+C Z_{1}(\ln \lambda) g_{M}\left(Z_{2}, \psi U\right)+\psi U(\ln \lambda)\left(g_{M}\left(Z_{1}, C Z_{2}\right)-g_{M}\left(Z_{2}, C Z_{1}\right)\right)\right\},
\end{aligned}
$$

ii) $\tilde{Q}\left\{B\left\{\mathcal{A}_{Z_{1}} B Z_{2}+h \stackrel{M}{\nabla} Z_{Z_{1}} C Z_{2}-\mathcal{A}_{Z_{2}} B Z_{1}-h \stackrel{M}{\nabla} Z_{Z_{2}} C Z_{1}\right\}\right\}=0$ 
are provided for any $Z_{1}, Z_{2} \in \Gamma\left(\left(k e r \Phi_{*}\right)^{\perp}\right), U \in \Gamma\left(\mathcal{D}_{\theta}\right)$ and $V \in \Gamma\left(\mathcal{D}_{\perp}\right)$.

Proof We search $g_{M}\left(\left[Z_{1}, Z_{2}\right], U\right)=0$ and $g_{M}\left(\left[Z_{1}, Z_{2}\right], V\right)=0$ for any $Z_{1}, Z_{2} \in \Gamma\left(\left(k e r \Phi_{*}\right)^{\perp}\right)$, $U \in \Gamma\left(\mathcal{D}_{\theta}\right)$ and $V \in \Gamma\left(\mathcal{D}_{\perp}\right)$. Firstly, using (10), (11) and (17), we get

$$
\begin{aligned}
g_{M}\left(\left[Z_{1}, Z_{2}\right], U\right) & =g_{M}\left(\mathcal{A}_{Z_{1}} B Z_{2}+h \stackrel{M}{\nabla} Z_{1} C Z_{2}-\mathcal{A}_{Z_{2}} B Z_{1}-h \stackrel{M}{\nabla} Z_{2} C Z_{1}, \psi U\right) \\
& +g_{M}\left(v \stackrel{M}{\nabla} Z_{1} B Z_{2}+\mathcal{A}_{Z_{1}} C Z_{2}-v \stackrel{M}{\nabla} Z_{Z_{2}} B Z_{1}-\mathcal{A}_{Z_{2}} C Z_{1}, \phi U\right) .
\end{aligned}
$$

We have $\left(\nabla \Phi_{*}\right)\left(Z_{1}, B Z_{2}\right)=-\Phi_{*}\left(\mathcal{A}_{Z_{1}} B Z_{2}\right)$ from (5) and equality of $\Phi_{*}\left(h \stackrel{M}{\nabla} Z_{1} C Z_{2}\right)$ from (13). In (27), we obtain

$$
\begin{aligned}
g_{M}\left(\left[Z_{1}, Z_{2}\right], U\right) & =\frac{1}{\lambda^{2}} g_{N}\left(\left(\nabla \Phi_{*}\right)\left(Z_{2}, B Z_{1}\right)-\left(\nabla \Phi_{*}\right)\left(Z_{1}, B Z_{2}\right), \Phi_{*}(\psi U)\right) \\
& +\frac{1}{\lambda^{2}} g_{N}\left(\nabla_{Z_{1}}^{\Phi} \Phi_{*}\left(C Z_{2}\right)-\nabla_{Z_{2}}^{\Phi} \Phi_{*}\left(C Z_{1}\right), \Phi_{*}(\psi U)\right) \\
& -Z_{1}(\ln \lambda) g_{M}\left(C Z_{2}, \psi U\right)-C Z_{2}(\ln \lambda) g_{M}\left(Z_{1}, \psi U\right) \\
& +\psi U(\ln \lambda) g_{M}\left(Z_{1}, C Z_{2}\right)+Z_{2}(\ln \lambda) g_{M}\left(C Z_{1}, \psi U\right) \\
& +C Z_{1}(\ln \lambda) g_{M}\left(Z_{2}, \psi U\right)-\psi U(\ln \lambda) g_{M}\left(Z_{2}, C Z_{1}\right) \\
& +g_{M}\left(v \stackrel{M}{\nabla} Z_{1} B Z_{2}+\mathcal{A}_{Z_{1}} C Z_{2}-v \stackrel{M}{Z_{2}} B Z_{1}-\mathcal{A}_{Z_{2}} C Z_{1}, \phi U\right) .
\end{aligned}
$$

We get (i) from (28). Now, for (10) and (11) we obtain

$$
\begin{aligned}
g_{M}\left(\left[Z_{1}, Z_{2}\right], V\right) & =-g_{M}\left(B\left\{\mathcal{A}_{Z_{1}} B Z_{2}+h \stackrel{M}{\nabla}{ }_{Z_{1}} C Z_{2}\right\}, V\right) \\
& +g_{M}\left(B\left\{\mathcal{A}_{Z_{2}} B Z_{1}+h \stackrel{M}{\nabla} Z_{2} C Z_{1}\right\}, V\right) .
\end{aligned}
$$

From (16) and (29), we get (ii).

In the rest of the section, we investigate totally geodesicity conditions on total manifold. Recall that $\Phi$ is said to be horizontally homothetic map if $h(\operatorname{grad}(\ln \lambda))=0$ [4] and $\Phi$ is said to be totally geodesic map if $\left(\nabla \Phi_{*}\right)(E, F)=0$ for all $E, F \in \Gamma(T M)$ [16].

Theorem 3.11 Let $\Phi$ be a conformal hemi-slant Riemannian map from a Kähler manifold $\left(M, g_{M}, J\right)$ to a Riemannian manifold $\left(N, g_{N}\right)$. Then, any two conditions below imply the third condition;

i) $\operatorname{ker} \Phi_{*}$ defines a totally geodesic foliation on $M$,

ii) $\Phi$ is a horizontally homothetic map, 
iii)

$$
\begin{aligned}
\stackrel{N}{\nabla^{\Phi}}{ }_{J W_{1}} \Phi_{*}\left(\psi W_{2}\right) & =\Phi_{*}\left(J\left[J W_{1}, W_{2}\right]\right)+\left(\nabla \Phi_{*}\right)^{\perp}\left(\psi W_{1}, \psi W_{2}\right) \\
& +\Phi_{*}\left(\mathcal{T}_{\phi W_{1}} \phi W_{2}+\mathcal{A}_{\psi W_{2}} \phi W_{1}+\mathcal{A}_{\psi W_{1}} \phi W_{2}\right)
\end{aligned}
$$

for any $W_{1}, W_{2} \in \Gamma\left(\operatorname{ker} \Phi_{*}\right)$.

Proof Using equations (5) and (13), we get

$$
\begin{aligned}
\Phi_{*}\left(\nabla_{J W_{1}}^{M} J W_{2}\right) & =\nabla^{\Phi}{ }_{J W_{1}} \Phi_{*}\left(J W_{2}\right)-\left(\nabla \Phi_{*}\right)\left(J W_{1}, J W_{2}\right) \\
& =\nabla^{N}{ }_{J W_{1}} \Phi_{*}\left(\psi W_{2}\right)-\Phi_{*}\left(\mathcal{T}_{\phi W_{1}} \phi W_{2}+\mathcal{A}_{\psi W_{2}} \phi W_{1}+\mathcal{A}_{\psi W_{1}} \phi W_{2}\right) \\
& -\psi W_{1}(\ln \lambda) \Phi_{*}\left(\psi W_{2}\right)-\psi W_{2}(\ln \lambda) \Phi_{*}\left(\psi W_{1}\right) \\
& +g_{M}\left(\psi W_{1}, \psi W_{2}\right) \Phi_{*}(\operatorname{grad}(\ln \lambda))-\left(\nabla \Phi_{*}\right)^{\perp}\left(\psi W_{1}, \psi W_{2}\right)
\end{aligned}
$$

for any $W_{1}, W_{2} \in \Gamma\left(\operatorname{ker} \Phi_{*}\right)$. On the other hand, we get

$$
\begin{aligned}
\Phi_{*}\left(\nabla_{J W_{1}}^{M} J W_{2}\right) & \left.=\Phi_{*}\left(J\left[J W_{1}, W_{2}\right]\right)+J \nabla_{W_{1}}^{M} J W_{2}\right) \\
& =\Phi_{*}\left(J\left[J W_{1}, W_{2}\right]\right)-\Phi_{*}\left(\stackrel{M}{\nabla}_{W_{1}} W_{2}\right) .
\end{aligned}
$$

Putting (31) in (30), we obtain

$$
\begin{aligned}
\Phi_{*}\left(\stackrel{M}{\nabla} W_{1} W_{2}\right) & =\Phi_{*}\left(J\left[J W_{1}, W_{2}\right]\right)-\nabla^{\Phi}{ }_{J W_{1}} \Phi_{*}\left(\psi W_{2}\right) \\
& +\Phi_{*}\left(\mathcal{T}_{\phi W_{1}} \phi W_{2}+\mathcal{A}_{\psi W_{2}} \phi W_{1}+\mathcal{A}_{\psi W_{1}} \phi W_{2}\right) \\
& +\psi W_{1}(\ln \lambda) \Phi_{*}\left(\psi W_{2}\right)+\psi W_{2}(\ln \lambda) \Phi_{*}\left(\psi W_{1}\right) \\
& -g_{M}\left(\psi W_{1}, \psi W_{2}\right) \Phi_{*}(\operatorname{grad}(\ln \lambda))+\left(\nabla \Phi_{*}\right)^{\perp}\left(\psi W_{1}, \psi W_{2}\right) .
\end{aligned}
$$

Suppose that (i) and (ii) are provided in (32). Then, we have

$$
\Phi_{*}\left(\nabla_{W_{1}}^{M} W_{2}\right)=0
$$

and

$$
\psi W_{1}(\ln \lambda) \Phi_{*}\left(\psi W_{2}\right)+\psi W_{2}(\ln \lambda) \Phi_{*}\left(\psi W_{1}\right)-g_{M}\left(\psi W_{1}, \psi W_{2}\right) \Phi_{*}(\operatorname{grad}(\ln \lambda))=0 .
$$

Hence, we obtain

$$
\begin{aligned}
0 & =\Phi_{*}\left(J\left[J W_{1}, W_{2}\right]\right)-\nabla^{N}{ }_{J W_{1}} \Phi_{*}\left(\psi W_{2}\right)+\left(\nabla \Phi_{*}\right)^{\perp}\left(\psi W_{1}, \psi W_{2}\right) \\
& +\Phi_{*}\left(\mathcal{T}_{\phi W_{1}} \phi W_{2}+\mathcal{A}_{\psi W_{2}} \phi W_{1}+\mathcal{A}_{\psi W_{1}} \phi W_{2}\right) .
\end{aligned}
$$


We get (iii) from (33). One can easily see that if (ii) and (iii) are provided in (32) we obtain $\Phi_{\star}\left(\stackrel{M}{\nabla} W_{1} W_{2}\right)=0$. So, (i) is satisfied. Lastly, we proof (ii). Suppose that (i) and (iii) are provided in (32). Then, we obtain

$$
\begin{aligned}
0 & =\psi W_{1}(\ln \lambda) \Phi_{*}\left(\psi W_{2}\right)+\psi W_{2}(\ln \lambda) \Phi_{*}\left(\psi W_{1}\right) \\
& -g_{M}\left(\psi W_{1}, \psi W_{2}\right) \Phi_{*}(\operatorname{grad}(\ln \lambda)) .
\end{aligned}
$$

For $\psi W_{1} \in \Gamma\left(\left(k e r \Phi_{*}\right)^{\perp}\right)$ in $(34)$, we have

$$
0=\lambda^{2} \psi W_{2}(\ln \lambda) g_{M}\left(\psi W_{1}, \psi W_{1}\right)
$$

So, we obtain $\psi W_{2}(\ln \lambda)=0$. It means $\lambda$ is a constant on $\left(\operatorname{ker} \Phi_{*}\right)^{\perp}$. Therefore, $\Phi$ is a horizontally homothetic map. The proof is complete.

In a similar way, we have the following.

Theorem 3.12 Let $\Phi$ be a conformal hemi-slant Riemannian map from a Kähler manifold $\left(M, g_{M}, J\right)$ to a Riemannian manifold $\left(N, g_{N}\right)$. Then, any two conditions below imply the third condition;

i) $\left(\operatorname{ker} \Phi_{*}\right)^{\perp}$ defines a totally geodesic foliation on $M$,

ii) $\Phi$ is a horizontally homothetic map,

iii)

$$
\begin{aligned}
\stackrel{N}{\nabla_{J Z_{1}}^{\Phi} \Phi_{*}\left(C Z_{2}\right)}= & \Phi_{*}\left(J\left[Z_{1}, J Z_{2}\right]\right)-\left(\nabla \Phi_{*}\right)^{\perp}\left(C Z_{1}, C Z_{2}\right) \\
& +\Phi_{*}\left(\mathcal{T}_{B Z_{1}} B Z_{2}+\mathcal{A}_{C Z_{1}} B Z_{2}+\mathcal{A}_{C Z_{2}} B Z_{1}\right)
\end{aligned}
$$

for any $Z_{1}, Z_{2} \in \Gamma\left(\left(\operatorname{ker} \Phi_{*}\right)^{\perp}\right)$.

Theorem 3.13 Let $\Phi$ be a conformal hemi-slant Riemannian map from a Kähler manifold $\left(M, g_{M}, J\right)$ to a Riemannian manifold $\left(N, g_{N}\right)$. Then, the slant distribution $\mathcal{D}_{\theta}$ defines a totally geodesic foliation on $M$ if and only if

$$
\cos ^{2} \theta \mathcal{T}_{U_{1}} U_{2}=\mathcal{T}_{U_{1}} B \psi U_{2}
$$

is provided for any $U_{1}, U_{2} \in \Gamma\left(\mathcal{D}_{\theta}\right)$. 
Proof From definition of $\nabla \Phi_{*}$ and (17), we get

$$
\begin{aligned}
\left(\nabla \Phi_{*}\right)\left(U_{1}, U_{2}\right) & =\Phi_{*}\left(\stackrel{M}{\nabla}{ }_{U_{1}} J U_{2}\right) \\
& =\Phi_{*}\left(\stackrel{M}{\nabla}_{U_{1}} J \phi U_{2}\right)+\Phi_{*}\left(\stackrel{M}{\nabla} U_{1} J \psi U_{2}\right) \\
& =\Phi_{*}\left(\stackrel{M}{\nabla} U_{1} \phi^{2} U_{2}+\stackrel{M}{\nabla} U_{1} \psi \phi U_{2}\right)+\Phi_{*}\left(\stackrel{M}{\nabla} U_{1} B \psi U_{2}+\stackrel{M}{\nabla} U_{1} C \psi U_{2}\right)
\end{aligned}
$$

for any $U_{1}, U_{2} \in \Gamma\left(\mathcal{D}_{\theta}\right)$. Now, from Theorem 3.8 and by using (20) in (34), we obtain

$$
\left(\nabla \Phi_{*}\right)\left(U_{1}, U_{2}\right)=-\cos ^{2} \theta \Phi_{*}\left(\mathcal{T}_{U_{1}} U_{2}\right)+\Phi_{*}\left(\mathcal{T}_{U_{1}} B \psi U_{2}\right)
$$

The proof is complete.

Theorem 3.14 Let $\Phi$ be a conformal hemi-slant Riemannian map from a Kähler manifold $\left(M, g_{M}, J\right)$ to a Riemannian manifold $\left(N, g_{N}\right)$. Then, any two conditions below imply the third condition;

i) $\mathcal{D}_{\perp}$ defines a totally geodesic foliation on $M$,

ii) $\lambda$ is a constant on $J\left(\mathcal{D}_{\perp}\right)$,

iii) $\stackrel{N}{\nabla^{\Phi}}{ }_{J V_{1}} \Phi_{*}\left(J V_{2}\right)=\left(\nabla \Phi_{*}\right)^{\perp}\left(J V_{1}, J V_{2}\right)-\Phi_{*}\left(J\left[V_{2}, J V_{1}\right]\right)$

for any $V_{1}, V_{2} \in \Gamma\left(\mathcal{D}_{\perp}\right)$.

Proof From the definition of $\nabla \Phi_{*}$, we have

$$
\begin{aligned}
& \left(\nabla \Phi_{*}\right)\left(J V_{1}, J V_{2}\right)=\stackrel{N}{\nabla^{\Phi}}{ }_{J V_{1}} \Phi_{*}\left(J V_{2}\right)-\Phi_{*}\left(\stackrel{M}{\nabla}{ }_{J V_{1}} J V_{2}\right) \\
& =\stackrel{N}{\nabla^{\Phi}}{ }_{J V_{1}} \Phi_{*}\left(J V_{2}\right)+\Phi_{*}\left(J\left[V_{2}, J V_{1}\right]-J \nabla_{V_{2}}^{M} J V_{1}\right) \\
& =\stackrel{N}{\nabla^{\Phi}}{ }_{J V_{1}} \Phi_{*}\left(J V_{2}\right)+\Phi_{*}\left(J\left[V_{2}, J V_{1}\right]\right)+\Phi_{*}\left(\stackrel{M}{\nabla} V_{2} V_{1}\right)
\end{aligned}
$$

for any $V_{1}, V_{2} \in \Gamma\left(\mathcal{D}_{\perp}\right)$. Using (13) in (37), we obtain

$$
\begin{aligned}
\Phi_{*}\left(\stackrel{M}{\nabla} V_{2} V_{1}\right) & =\stackrel{N}{N}{ }_{J V_{1}} \Phi_{\star}\left(J V_{2}\right)-\Phi_{*}\left(J\left[V_{2}, J V_{1}\right]\right) \\
& +J V_{1}(\ln \lambda) \Phi_{*}\left(J V_{2}\right)+J V_{2}(\ln \lambda) \Phi_{*}\left(J V_{1}\right) \\
& -g_{M}\left(J V_{1}, J V_{2}\right) \Phi_{*}(\operatorname{grad}(\ln \lambda))+\left(\nabla \Phi_{*}\right)^{\perp}\left(J V_{1}, J V_{2}\right) .
\end{aligned}
$$

Suppose that (i) and (iii) are satisfies in (38). So, we have

$$
0=\Phi_{*}\left(\stackrel{M}{\nabla} V_{V_{2}} V_{1}\right)
$$


and

$$
0=-\nabla_{J V_{1}}^{N} \Phi_{*}\left(J V_{2}\right)-\Phi_{*}\left(J\left[V_{2}, J V_{1}\right]\right)+\left(\nabla \Phi_{*}\right)^{\perp}\left(J V_{1}, J V_{2}\right) .
$$

Therefore, we obtain from (38)

$$
\begin{aligned}
0 & =J V_{1}(\ln \lambda) \Phi_{*}\left(J V_{2}\right)+J V_{2}(\ln \lambda) \Phi_{*}\left(J V_{1}\right) \\
& -g_{M}\left(J V_{1}, J V_{2}\right) \Phi_{*}(\operatorname{grad}(\ln \lambda)) .
\end{aligned}
$$

Now, we obtain from (39)

$$
0=\lambda^{2} J V_{2}(\ln \lambda) g_{M}\left(J V_{1}, J V_{1}\right)
$$

for any $V_{1} \in \Gamma\left(\mathcal{D}_{\perp}\right)$. So, we obtain $J V_{2}(\ln \lambda)=0$. It means $\lambda$ is a constant on $J\left(\mathcal{D}_{\perp}\right)$. The proofs of (i) and (iii) are easy to see from (38).

Lastly, we present totally geodesicity of the map $\Phi$.

Theorem 3.15 Let $\Phi$ be a conformal hemi-slant Riemannian map from a Kähler manifold $\left(M, g_{M}, J\right)$ to a Riemannian manifold $\left(N, g_{N}\right)$. Then, the map $\Phi$ defines a totally geodesic foliation on $M$ if and only if

i) $\Phi$ is a horizontally homothetic map,

ii)

$$
\begin{aligned}
\nabla_{Z_{1}}^{N} \Phi_{*}\left(Z_{2}\right) & =\left(\nabla \Phi_{*}\right)^{\perp}\left(Z_{1}, Z_{2}\right)-\Phi_{*}\left(C\left\{\mathcal{A}_{Z_{1}} B Z_{2}+h{\stackrel{M}{\nabla} Z_{1}} C Z_{2}\right\}\right) \\
& -\Phi_{*}\left(\psi\left\{v \stackrel{M}{\nabla} Z_{1} B Z_{2}+\mathcal{A}_{Z_{1}} C Z_{2}\right\}\right),
\end{aligned}
$$

iii)

$$
\begin{aligned}
\cos ^{2} \theta \mathcal{T}_{W_{1}} \tilde{P} W_{2} & =h \nabla_{W_{1}}^{M} \phi \tilde{P} W_{2}+\psi \mathcal{T}_{W_{1}}\left(\psi \tilde{P} W_{2}+J \tilde{Q} W_{2}\right) \\
& +C h \nabla_{W_{1}}\left(\psi \tilde{P} W_{2}+J \tilde{Q} W_{2}\right)
\end{aligned}
$$

are provided for any $Z_{1}, Z_{2} \in \Gamma\left(\left(k e r \Phi_{*}\right)^{\perp}\right)$ and $W_{1}, W_{2} \in \Gamma\left(k e r \Phi_{*}\right)$.

Proof Because of rank condition of the conformal hemi-slant Riemannian map $\Phi$, we have $\left(\nabla \Phi_{*}\right)\left(Z_{1}, Z_{2}\right)=\left(\nabla \Phi_{*}\right)^{\perp}\left(Z_{1}, Z_{2}\right)+\left(\nabla \Phi_{*}\right)^{\top}\left(Z_{1}, Z_{2}\right)$ for any $Z_{1}, Z_{2} \in \Gamma\left(\left(k e r \Phi_{*}\right)^{\perp}\right)$. We know that $\left(\nabla \Phi_{*}\right)^{\perp}\left(Z_{1}, Z_{2}\right) \in \Gamma\left(\left(\operatorname{range} \Phi_{*}\right)^{\perp}\right)$ and $\left(\nabla \Phi_{*}\right)^{\top}\left(Z_{1}, Z_{2}\right) \in \Gamma\left(\operatorname{range} \Phi_{*}\right)$, see (12) and (13). Using these equations, we obtain

$$
\left(\nabla \Phi_{*}\right)\left(Z_{1}, Z_{2}\right)=\stackrel{N}{\nabla^{\Phi}} Z_{1} \Phi_{*}\left(Z_{2}\right)-\Phi_{*}\left(\nabla_{Z_{1}} Z_{2}\right) .
$$


Since $\left(\nabla \Phi_{*}\right)\left(Z_{1}, Z_{2}\right)=0$,

$$
\begin{aligned}
& 0=\stackrel{N}{\nabla^{\Phi}}{ }_{Z_{1}} \Phi_{*}\left(Z_{2}\right)-\left(\nabla \Phi_{*}\right)^{\perp}\left(Z_{1}, Z_{2}\right) \\
& +\Phi_{*}\left(C \mathcal{A}_{Z_{1}} B Z_{2}+\psi v v_{Z_{1}}^{M} B Z_{2}\right) \\
& +\Phi_{*}\left(\psi \mathcal{A}_{Z_{1}} C Z_{2}+C h \stackrel{M}{\nabla} Z_{1} C Z_{2}\right) \\
& \text { - } Z_{1}(\ln \lambda) \Phi_{*}\left(Z_{2}\right)-Z_{2}(\ln \lambda) \Phi_{*}\left(Z_{1}\right) \\
& +g_{M}\left(Z_{1}, Z_{2}\right) \Phi_{*}(\operatorname{grad}(\ln \lambda)) \text {. }
\end{aligned}
$$

From (42), we have

$$
\begin{aligned}
\stackrel{N}{\nabla^{\Phi} Z_{1} \Phi_{*}\left(Z_{2}\right)} & =\left(\nabla \Phi_{*}\right)^{\perp}\left(Z_{1}, Z_{2}\right)-\Phi_{*}\left(C\left\{\mathcal{A}_{Z_{1}} B Z_{2}+h{\stackrel{M}{\nabla} Z_{1}} C Z_{2}\right\}\right) \\
& -\Phi_{*}\left(\psi\left\{v \stackrel{M}{\nabla} Z_{1} B Z_{2}+\mathcal{A}_{Z_{1}} C Z_{2}\right\}\right)
\end{aligned}
$$

and

$$
\begin{aligned}
0 & =Z_{1}(\ln \lambda) \Phi_{*}\left(Z_{2}\right)+Z_{2}(\ln \lambda) \Phi_{*}\left(Z_{1}\right) \\
& -g_{M}\left(Z_{1}, Z_{2}\right) \Phi_{*}(\operatorname{grad}(\ln \lambda)) .
\end{aligned}
$$

In (44), for any $Z_{1} \in \Gamma\left(\left(k e r \Phi_{*}\right)^{\perp}\right)$ we get

$$
\begin{aligned}
0 & =\lambda^{2} Z_{1}(\ln \lambda) g_{M}\left(Z_{2}, Z_{1}\right)+\lambda^{2} Z_{2}(\ln \lambda) g_{M}\left(Z_{1}, Z_{1}\right) \\
& -\lambda^{2} g_{M}\left(Z_{1}, Z_{2}\right) Z_{1}(\ln \lambda) \\
& =\lambda^{2} Z_{2}(\ln \lambda) g_{M}\left(Z_{1}, Z_{1}\right) .
\end{aligned}
$$

So, from (45) we obtain $Z_{2}(\ln \lambda)=0$. It means $\Phi$ is a horizontally homothetic map. We obtain (ii) and (i) from (43) and (45), respectively. In a similar way, we get

$$
\begin{aligned}
\left(\nabla \Phi_{*}\right)\left(W_{1}, W_{2}\right) & =\Phi_{*}\left(J^{M} \nabla_{W_{1}} J \tilde{P} W_{2}+J \tilde{Q} W_{2}\right) \\
& =\Phi_{*}\left(\nabla_{W_{1}} \phi^{2} \tilde{P} W_{2}+\stackrel{M}{\nabla} W_{1} \psi \phi \tilde{P} W_{2}\right) \\
& +\Phi_{*}\left(\psi \mathcal{T}_{W_{1}} \psi \tilde{P} W_{2}+C h \nabla_{W_{1}}^{M} \tilde{P} W_{2}\right) \\
& +\Phi_{*}\left(\psi \mathcal{T}_{W_{1}} J \tilde{Q} W_{2}+C h \nabla_{W_{1}}^{M} J \tilde{Q} W_{2}\right) \\
& =-\cos ^{2} \theta \Phi_{*}\left(\mathcal{T}_{W_{1}} \tilde{P} W_{2}\right)+\Phi_{*}\left(h \nabla_{W_{1}} \psi \phi \tilde{P} W_{2}\right) \\
& +\Phi_{*}\left(\psi \mathcal{T}_{W_{1}}\left(\psi \tilde{P} W_{2}+J \tilde{Q} W_{2}\right)\right) \\
& +\Phi_{*}\left(C h{\stackrel{M}{W_{1}}}\left(\psi \tilde{P} W_{2}+J \tilde{Q} W_{2}\right)\right)
\end{aligned}
$$


for any $W_{1}, W_{2} \in \Gamma\left(\operatorname{ker} \Phi_{*}\right)$. We obtain (iii) from (46). The proof is complete.

\section{Pluriharmonic Conformal Hemi-slant Riemannian Maps}

In this section, we use the notion of pluriharmonic map on the distributions of a conformal hemislant Riemannian map to introduce their geometric properties. $\Phi$ is said to be $\mathcal{D}_{\theta}$-pluriharmonic $\operatorname{map}\left(\mathcal{D}_{\perp}, \operatorname{ker} \Phi_{*},\left(\operatorname{ker} \Phi_{*}\right)^{\perp}\right.$ or mixed-pluriharmonic, respectively) if

$$
\left(\nabla \Phi_{*}\right)(E, F)+\left(\nabla \Phi_{*}\right)(J E, J F)=0
$$

for $E, F \in \Gamma\left(\mathcal{D}_{\theta}\right)\left(\mathcal{D}_{\perp}, \operatorname{ker} \Phi_{*},\left(\operatorname{ker} \Phi_{*}\right)^{\perp}\right.$ or $\left(\operatorname{ker} \Phi_{*}\right)^{\perp} \times \operatorname{ker} \Phi_{*}$, respectively) [19].

Theorem 4.1 Let $\Phi$ be a conformal hemi-slant Riemannian map from a Kähler manifold $\left(M, g_{M}, J\right)$ to a Riemannian manifold $\left(N, g_{N}\right)$. Then, any two conditions below imply the third condition;

i) $\Phi$ is a $\mathcal{D}_{\theta}$-pluriharmonic map,

ii) $\lambda$ is a constant on $\psi\left(\mathcal{D}_{\theta}\right)$ and $\left(\nabla \Phi_{*}\right)^{\perp}\left(\psi U_{1}, \psi U_{2}\right)=0$,

iii) $\sin ^{2} \theta \mathcal{T}_{U_{1}} U_{2}+\mathcal{A}_{\psi U_{2}} \phi U_{1}+\mathcal{A}_{\psi U_{1}} \phi U_{2}=0$

for any $U_{1}, U_{2} \in \Gamma\left(\mathcal{D}_{\theta}\right)$.

Proof Using definition of $\mathcal{D}_{\theta}$-pluriharmonic map and symmetry condition of $\nabla \Phi_{*}$, we get

$$
\begin{aligned}
0 & =\left(\nabla \Phi_{*}\right)\left(U_{1}, U_{2}\right)+\left(\nabla \Phi_{*}\right)\left(\phi U_{1}, \phi U_{2}\right)+\left(\nabla \Phi_{*}\right)\left(\psi U_{2}, \phi U_{1}\right) \\
& +\left(\nabla \Phi_{*}\right)\left(\psi U_{1}, \phi U_{2}\right)+\left(\nabla \Phi_{*}\right)\left(\psi U_{1}, \psi U_{2}\right)
\end{aligned}
$$

for any $U_{1}, U_{2} \in \Gamma\left(\mathcal{D}_{\theta}\right)$. From Theorem 3.8 and (12), we obtain

$$
\begin{aligned}
0 & =-\sin ^{2} \theta \Phi_{*}\left(\mathcal{T}_{U_{1}} U_{2}\right)-\Phi_{*}\left(\mathcal{A}_{\psi U_{2}} \phi U_{1}+\mathcal{A}_{\psi U_{1}} \phi U_{2}\right) \\
& +\psi U_{1}(\ln \lambda) \Phi_{*}\left(\psi U_{2}\right)+\psi U_{2}(\ln \lambda) \Phi_{*}\left(\psi U_{1}\right) \\
& -g_{M}\left(\psi U_{1}, \psi U_{2}\right) \Phi_{*}(\operatorname{grad}(\ln \lambda))-\left(\nabla \Phi_{*}\right)^{\perp}\left(\psi U_{1}, \psi U_{2}\right)
\end{aligned}
$$

Now, suppose that (i) and (ii) are provided in (48). So, we have

$$
\begin{gathered}
\left(\nabla \Phi_{*}\right)\left(U_{1}, U_{2}\right)+\left(\nabla \Phi_{\star}\right)\left(J U_{1}, J U_{2}\right)=0 \\
\psi U_{1}(\ln \lambda) \Phi_{\star}\left(\psi U_{2}\right)+\psi U_{2}(\ln \lambda) \Phi_{*}\left(\psi U_{1}\right)-g_{M}\left(\psi U_{1}, \psi U_{2}\right) \Phi_{\star}(\operatorname{grad}(\ln \lambda))=0
\end{gathered}
$$

and

$$
\left(\nabla \Phi_{*}\right)^{\perp}\left(\psi U_{1}, \psi U_{2}\right)=0
$$


respectively. Hence, we easily obtain (iii) from (48). If we suppose that (ii) and (iii) are provided in (48), we obtain (i) from (47) such that $\Phi$ is a $\mathcal{D}_{\theta}$-pluriharmonic map. Lastly, we suppose that (i) and (iii) are provided in (48), we get

$$
\begin{aligned}
0 & =\psi U_{1}(\ln \lambda) \Phi_{*}\left(\psi U_{2}\right)+\psi U_{2}(\ln \lambda) \Phi_{*}\left(\psi U_{1}\right) \\
& -g_{M}\left(\psi U_{1}, \psi U_{2}\right) \Phi_{*}(\operatorname{grad}(\ln \lambda))-\left(\nabla \Phi_{*}\right)^{\perp}\left(\psi U_{1}, \psi U_{2}\right)
\end{aligned}
$$

We obtain $\left(\nabla \Phi_{*}\right)^{\perp}\left(\psi U_{1}, \psi U_{2}\right)=0$ from (49). For any $\psi U_{1} \in \Gamma\left(\psi\left(\mathcal{D}_{\theta}\right)\right)$, we obtain

$$
0=\lambda^{2} \psi U_{2}(\ln \lambda) g_{M}\left(\psi U_{1}, \psi U_{1}\right)
$$

So, from $(50)$ we get $\psi U_{2}(\ln \lambda)=0$. It means $\lambda$ is a constant on $\psi\left(\mathcal{D}_{\theta}\right)$. (ii) is provided. The proof is all.

Similarly, we have the following theorems.

Theorem 4.2 Let $\Phi$ be a conformal hemi-slant Riemannian map from a Kähler manifold $\left(M, g_{M}, J\right)$ to a Riemannian manifold $\left(N, g_{N}\right)$. Then, any two conditions below imply the third condition;

i) $\mathcal{D}_{\perp}$ defines a totally geodesic foliation on $M$,

ii) $\Phi$ is a $\mathcal{D}_{\perp}$-pluriharmonic map,

iii) $\lambda$ is a constant on $J\left(\mathcal{D}_{\perp}\right)$ and $\left(\nabla \Phi_{*}\right)^{\perp}\left(J V_{1}, J V_{2}\right)=0$

for any $V_{1}, V_{2} \in \Gamma\left(\mathcal{D}_{\perp}\right)$.

Note that $\mathcal{D}_{\perp}$-pluriharmonic map and $J\left(\mathcal{D}_{\perp}\right)$-pluriharmonic map give same results for a conformal hemi-slant Riemannian map. Since $\mathcal{D}_{\perp}$ is an anti-invariant distribution, we obtain the result from the definition of pluriharmonic map.

Theorem 4.3 Let $\Phi$ be a conformal hemi-slant Riemannian map from a Kähler manifold $\left(M, g_{M}, J\right)$ to a Riemannian manifold $\left(N, g_{N}\right)$. Then, any two conditions below imply the third condition;

i) $\Phi$ is a $\operatorname{ker} \Phi_{*}$-pluriharmonic map,

ii) $\Phi$ is a horizontally homothetic map and $\left(\nabla \Phi_{*}\right)^{\perp}\left(\psi W_{1}, \psi W_{2}\right)=0$,

iii) $\sin ^{2} \theta \mathcal{T}_{W_{1}} W_{2}=\mathcal{A}_{\psi W_{2}} \phi W_{1}+\mathcal{A}_{\psi W_{1}} \phi W_{2}$

for any $W_{1}, W_{2} \in \Gamma\left(\operatorname{ker} \Phi_{*}\right)$.

Theorem 4.4 Let $\Phi$ be a conformal hemi-slant Riemannian map from a Kähler manifold $\left(M, g_{M}, J\right)$ to a Riemannian manifold $\left(N, g_{N}\right)$. Then, any three conditions below imply the fourth condition; 
i) $\left(\operatorname{ker} \Phi_{*}\right)^{\perp}$ defines a totally geodesic foliation on $M$,

ii) $\Phi$ is a $\left(\operatorname{ker} \Phi_{*}\right)^{\perp}$-pluriharmonic map,

iii) $\Phi$ is a horizontally homothetic map,

iv) $\stackrel{N}{\nabla^{\Phi}} Z_{1} \Phi_{\star}\left(Z_{2}\right)=\Phi_{\star}\left(\mathcal{T}_{B Z_{1}} B Z_{2}+\mathcal{A}_{C Z_{1}} B Z_{2}+\mathcal{A}_{C Z_{2}} B Z_{1}\right)+\left(\nabla \Phi_{\star}\right)^{\perp}\left(C Z_{1}, C Z_{2}\right)$

for any $Z_{1}, Z_{2} \in \Gamma\left(\left(\operatorname{ker} \Phi_{*}\right)^{\perp}\right)$.

Theorem 4.5 Let $\Phi$ be a conformal hemi-slant Riemannian map from a Kähler manifold $\left(M, g_{M}, J\right)$ to a Riemannian manifold $\left(N, g_{N}\right)$. Then, any two conditions below imply the third condition;

i) $\Phi$ is a mixed-pluriharmonic map,

ii) $\Phi$ is a horizontally homothetic map and $\left(\nabla \Phi_{*}\right)^{\perp}(C Z, \psi W)=0$,

iii) $\mathcal{A}_{Z} W+\mathcal{T}_{B Z} \phi W+\mathcal{A}_{\psi W} B Z+\mathcal{A}_{C Z} \phi W=0$

for any $Z \in \Gamma\left(\left(\operatorname{ker} \Phi_{*}\right)^{\perp}\right)$ and $W \in \Gamma\left(\operatorname{ker} \Phi_{\star}\right)$.

Proof From definition of mixed-pluriharmonic map, we obtain

$$
\begin{aligned}
0 & =-\Phi_{*}\left(\mathcal{A}_{Z} W\right)+\left(\nabla \Phi_{*}\right)^{\perp}(C Z, \psi W) \\
& -\Phi_{*}\left(\mathcal{T}_{B Z} \phi W+\mathcal{A}_{\psi W} B Z+\mathcal{A}_{C Z} \phi W\right) \\
& +C Z(\ln \lambda) \Phi_{*}(\psi W)+\psi W(\ln \lambda) \Phi_{*}(C Z) \\
& -g_{M}(C Z, \psi W) \Phi_{*}(\operatorname{grad}(\ln \lambda))
\end{aligned}
$$

for any $Z \in \Gamma\left(\left(\operatorname{ker} \Phi_{*}\right)^{\perp}\right)$ and $W \in \Gamma\left(\operatorname{ker} \Phi_{*}\right)$. Now, we only proof (ii). Suppose that (i) and (iii) are provided in (51). We obtain easily $\left(\nabla \Phi_{*}\right)^{\perp}(C Z, \psi W)=0$ and get

$$
0=\lambda^{2} \psi W(\ln \lambda) g_{M}(C Z, C Z)
$$

for $C Z \in \Gamma\left(\left(\operatorname{ker} \Phi_{*}\right)^{\perp}\right)$ and

$$
0=\lambda^{2} C Z(\ln \lambda) g_{M}(\psi W, \psi W)
$$

for $\psi W \in \Gamma\left(\left(\operatorname{ker} \Phi_{*}\right)^{\perp}\right)$. So, we have $\psi W(\ln \lambda)=0$ and $C Z(\ln \lambda)=0$ from (52) and (53), respectively. They means $\lambda$ is a constant on horizontal distribution. Hence, $\Phi$ is a horizontally homothetic map. (ii) is provided.

\section{Declaration of Ethical Standards}

The author declares that the materials and methods used in their study do not require ethical committee and/or legal special permission. 


\section{Conflict of Interest}

The author declares no conflicts of interest.

\section{References}

[1] Akyol M.A., Şahin B., Conformal anti-invariant submersions from almost Hermitian manifolds, Turkish Journal of Mathematics, 40, 43-70, 2016.

[2] Akyol M.A., Şahin B., Conformal semi-invariant submersions, Communications in Contemporary Mathematics, 19(2), 1650011, 2017.

[3] Akyol M.A., Şahin B., Conformal slant submersions, Hacettepe Journal of Mathematics and Statistics, 48, 28-44, 2019.

[4] Baird P., Wood J.C., Harmonic Morphisms between Riemannian Manifolds, Clarendon Press, 2003.

[5] Chen B.Y., Riemannian Submanifolds: Handbook of Differential Geometry, Vol. I, 187-418, 2000.

[6] Falcitelli M., Ianus S., Pastore A.M., Riemannian Submersions and Related Topics, World Scientific, 2004.

[7] Fischer A.E., Riemannian maps between Riemannian manifolds, Contemporary Mathematics, 132, 331-366, 1992.

[8] Garcia-Rio E., Kupeli D.N., Semi-Riemannian Maps and Their Applications, Kluwer Academic, 1999.

[9] Kumar S., Kumar S., Pandey S., Prasad R., Conformal hemi-slant submersions from almost Hermitian manifolds, Communications of the Korean Mathematical Society, 35(3), 999-1018, 2020.

[10] Nore T., Second fundamental form of a map, Annali di Matematica Pura ed Applicata, 146, 281-310, 1986.

[11] Ohnita Y., On pluriharmonicity of stable harmonic maps, Journal of the London Mathematical Society, 2(35), 563-587, 1987.

[12] O'Neill B., The fundamental equations of a submersion, Michigan Mathematical Journal, 13(4), 458469, 1966.

[13] Şahin B., Semi-invariant Riemannian maps from almost Hermitian manifolds, Indagationes Mathematicae, 23, 80-94, 2012.

[14] Şahin B., Slant Riemannian maps from almost Hermitian manifolds, Quaestiones Mathematicae, 36(3), 449-461, 2013.

[15] Şahin B., Hemi-slant Riemannian maps, Mediterranean Journal of Mathematics, 14, 2017.

[16] Şahin B., Riemannian Submersions, Riemannian Maps in Hermitian Geometry and Their Applications, Elsevier, 2017.

[17] Şahin B., Conformal Riemannian maps between Riemannian manifolds, their harmonicity and decomposition theorems, Acta Applicandae Mathematicae, 109(3), 829-847, 2010.

[18] Şahin B., Yanan Ş., Conformal Riemannian maps from almost Hermitian manifolds, Turkish Journal of Mathematics, 42(5), 2436-2451, 2018.

[19] Şahin B., Yanan Ş., Conformal semi-invariant Riemannian maps from almost Hermitian manifolds, Filomat, 33(4), 1125-1134, 2019.

[20] Yanan Ş., Conformal generic Riemannian maps from almost Hermitian manifolds, Turkish Journal of Science, 6(2), 76-88, 2021. 
[21] Yanan Ş., Şahin B., Conformal slant Riemannian maps, International Journal of Maps in Mathematics, 2022 (Accepted).

[22] Yano K., Kon M., Structures on Manifolds: Series in Pure Mathematics, World Scientific, 1984. 